\title{
Second chromosome polymorphism of Drosophila buzzatii in a natural population is not associated with gametic selection and does not affect mating pattern
}

\author{
C Rodriguez ${ }^{1 *}$, E Hasson $^{1}$, JJ Fanara ${ }^{1}, \mathrm{JC}_{\text {Vilardi }}{ }^{2}$ \\ ${ }^{1}$ GIBE, Universidad de Buenos Aires, Facultad de Ciencias Exactas y Naturales, \\ Depto Cs Biológicas, Ciudad Universitaria Pab II, 1428 Buenos Aires; \\ 2 Universidad de Buenos Aires, Facultad de Ciencias Exactas y Naturales, \\ Depto Cs Biológicas, Laboratorio de Genetica, Ciudad Universitaria Pab II, \\ 1428 Buenos Aires, Argentina
}

(Received 31 January 1991; accepted 13 January 1992)

\begin{abstract}
Summary - In previous work we have demonstrated the adaptive significance of the chromosomal polymorphism of $D$ buzzatii in a natural population from Argentina by means of selection components analysis. The experimental design employed for this purpose was based on 4 general assumptions. The results reported in the present study allow us to verify 3 of these assumptions: i) mating is at random, irrespective of the flies karyotype; ii) gametic selection may be considered absent; and iii) there is no differential attraction of flies to the collecting baits. The validity of these assumptions gives strong support to the conclusions reached in our previous selection components analysis.
\end{abstract}

mating pattern / gametic selection / chromosomal polymorphism

Résumé - Le polymorphisme du deuxième chromosome de Drosophila buzzatii dans une population naturelle n'est pas associé à une sélection gamétique et n'affecte pas le mode d'accouplement. Dans un travail précédent, nous avons démontré la signification adaptative du polymorphisme chromosomique de $\mathrm{D}$ buzzatii dans une population naturelle d'Argentine, à l'aide d'une analyse de la sélection en ses composantes. Le plan d'expérience utilisé dans ce but était basé sur quatre hypothèses. Les résultats présentés dans le présent travail permettent la vérification de trois d'entre elles: i) l'accouplement se produit au hasard, indépendamment du karyotype des mouches; ii) la sélection gamétique est absente; iii) il n'y a pas d'attraction différentielle des mouches vers les pièges de récolte. La

* Correspondence and reprints 
vérification de ces hypothèses confirme les conclusions établies dans notre précédente analyse des composantes de la sélection.

mode d'accouplement / sélection gamétique / polymorphisme chromosomique / drosophile

\section{INTRODUCTION}

Several methodological approaches have been applied to the study of natural selection in nature (see Endler, 1986 for a review). One of them consists of dividing total selection into partial components throughout the life-cycle (selection components analysis) (Hedrick and Murray, 1983). This approach was employed to investigate the adaptive significance of chromosomal polymorphisms in wild populations of the cactophilic fly Drosophila buzzatii (Ruiz et al, 1986; Hasson et al, 1991). These authors demonstrated not only the adaptive significance of the second chromosomal polymorphisms, but also that certain chromosomal arrangements are favoured during one selective episode and selected against in another, a pattern called endocyclic selection. Moreover, they suggested that this pattern might be involved in the maintenance of polymorphisms. However, their conclusions are based on the validity of 4 assumptions; i), random mating; ii), absence of gametic selection; iii), lack of differential attraction of flies to the collecting baits; and iv), lack of selection in the laboratory (see Ruiz et al, 1986).

In the present work we report the results of the analysis of the first 3 assumptions, with the aim of providing experimental support for our previous results.

\section{MATERIAL AND METHODS}

$D$ buzzatii is a cactophilic species that breeds and feeds on the decaying cladodes of several Opuntia species. The present work was performed in the natural population of Arroyo Escobar, where $D$ buzzatii breeds on the rotting cladodes of Opuntia vulgaris (for a description of this locality, see Hasson et al, 1991). Wild flies were caught in May 1987 by net sweeping on fermented banana baits. Flies were immediately sexed and females were maintained in individual vials with David's (1962) killed yeast culture medium at $25^{\circ} \mathrm{C}$.

The analyses of 8 larvae from the progeny of each wild inseminated female allowed us to assess the karyotype for the second chromosome of both parents assuming that the progeny of each female was fathered by a single male. Three chromosome arrangements were identified: st (standard), $2 j$ and $2 j z^{3}$. Observed mating frequencies were compared with those expected by random mating by means of $\chi^{2}$ tests.

Goodness of fit to Mendelian proportions was studied by means of $\chi^{2}$ tests for each mating class. Since the observed karyotypic proportions in some mating classes departed from the expected (see below), the segregation of $2 j / s t$ and $2 j z^{3} / s t$ heterokaryotypes was studied in more detail. This analysis was performed with heterozygous individuals obtained by crossing flies from homozygous stocks. 
These stocks were obtained through consanguineous crosses among the progeny of several wild females. Seventeen lines homozygous for $2 j, 14$ for $2 j z^{3}$ and 4 for $2 s t$ were isolated, and individually maintained until employed in further experiments. Heterokaryotypes were produced through both reciprocal crosses by setting together 100 mature virgin females and 100 mature males of the corresponding homozygous stocks in an egg collecting chamber. Heterozygous eggs were seeded in culture bottles in uncrowded conditions (6-8 eggs per ml of culture medium).

One hundred heterokaryotypic 5-day-old flies were crossed with 100 flies of each of the 3 homokaryotypic stocks. Flies were introduced into an egg collecting chamber for a $48 \mathrm{~h}$ period. The collecting medium was changed every $24 \mathrm{~h}$. In all cases, both reciprocal crosses were made.

Eggs were allowed to hatch and 500 first instar larvae were seeded in 5 culture bottles (100 larvae each) containing $40 \mathrm{cc}$ of culture medium for each cross, in low density conditions and maintained at $25^{\circ} \mathrm{C}$. Thirty-two third instar larvae from each bottle were sampled and their salivary gland chromosomes analyzed, according to Fontdevila et al (1981). Observed karyotypic proportions in the offspring of each cross were compared to those expected by means of goodness of fit $\chi^{2}$ tests. The remaining individuals in the bottles were maintained until pupation in order to estimate larval viability by counting all pupae.

Further collections were performed in A Escobar in March 1989 by means of banana baits and with Opuntia vulgaris rotting cladodes, in order to test the assumption that flies were not differentially attracted to the collecting baits.

\section{RESULTS}

The frequencies estimated for second chromosome arrangements were: standard $(s t)=0.110 ; j=0.575$ and $j z^{3}=0.315$. The observed and random mating expected numbers of each mating class are shown in table I. Since the frequencies of certain mating classes were extremely low, goodness of fit tests were performed for each chromosomal arrangement. None of the $\chi^{2}$ values was significant (table II).

Additionally, Mendelian segregation was tested for the most common mating classes. The results shown in table III revealed significant departures in crosses $j / s t \times j / j ; j / s t \times j / j z^{3}$ and $j z^{3} / s t \times j / j$. From this analysis it may be concluded that a deficiency of $2 s t$ bearing individuals occurred in all cases. These results could suggest gametic selection against $2 s t$. To test this hypothesis, flies heterozygous for $2 s t$ were backcrossed with individuals from the 3 homokaryotypic stocks. Significant departures from expected values were detected only in 2 independent crosses of $2 j / s t$ males with $2 s t / s t$ females (table IV, fourth row) with homogeneous results $\left(\chi^{2}=0.09, d f=1 P>0.75\right)$. This bias cannot be attributed to selection in the laboratory because there was no relationship between viability (table IV, fifth column) and segregation distortion for each cross. The results of the reciprocal cross did not depart from the expected values and when pooling the progeny that received $2 s t$ versus $2 j$ in all crosses involving the $2 s t / j$ heterokaryotype (from table IV), the $\chi^{2}$ value was not significant $\left(\chi^{2}=3.13, d f=1, P>0.05\right)$. Likewise, none of the crosses involving the $2 s t / j z^{3}$ parents showed a significant distortion in the segregation pattern. However, all crosses involving heterozygous $2 s t / *$ parental males showed a homogeneous trend $\left(\chi^{2}=5.03, d f=5, P>0.25\right)$ of $2 s t$ deficiency, 
Table I. Observed numbers of wild mating pairs sampled at Arroyo Escobar. Rows and columns stand for parental second chromosome karyotypes of Drosophila buzzatii. Numbers expected by random mating are shown in parentheses.

\begin{tabular}{|c|c|c|c|c|c|c|}
\hline & $j / j$ & $j / s t$ & $j / j z^{3}$ & $s t / s t$ & $s t / j z^{3}$ & $j z^{3} / j z^{3}$ \\
\hline$j / j$ & $\begin{array}{c}17 \\
(19.01)\end{array}$ & $\begin{array}{c}11 \\
(14.0)\end{array}$ & $\begin{array}{c}57 \\
(47.92)\end{array}$ & $\begin{array}{c}2 \\
(1.24)\end{array}$ & $\begin{array}{c}8 \\
(9.85)\end{array}$ & $\begin{array}{c}9 \\
(8.77)\end{array}$ \\
\hline$j / s t$ & & $\begin{array}{c}3 \\
(2.60)\end{array}$ & $\begin{array}{c}18 \\
(17.50)\end{array}$ & $\begin{array}{c}0 \\
(0.21)\end{array}$ & $\begin{array}{c}2 \\
(3.69)\end{array}$ & $\begin{array}{c}7 \\
(3.19)\end{array}$ \\
\hline$j / j z^{3}$ & & & $\begin{array}{c}23 \\
(30.41)\end{array}$ & $\begin{array}{c}2 \\
(1.64)\end{array}$ & $\begin{array}{c}15 \\
(12.31)\end{array}$ & $\begin{array}{c}11 \\
(11.30)\end{array}$ \\
\hline$s t / s t$ & & & & $\begin{array}{c}0 \\
(0.03)\end{array}$ & $\begin{array}{c}0 \\
(0.14)\end{array}$ & $\begin{array}{c}0 \\
(0.17)\end{array}$ \\
\hline$s t / j z^{3}$ & & & & & $\begin{array}{c}2 \\
(0.69)\end{array}$ & $\begin{array}{c}2 \\
(1.61)\end{array}$ \\
\hline$j z^{3} / j z^{3}$ & & & & & & $\begin{array}{c}0 \\
(0.94)\end{array}$ \\
\hline
\end{tabular}

and when the progeny of these crosses was pooled according to the arrangement received from their parental males: $\operatorname{st}(N=413)$ and non $s t(N=488)$ a significant distortion from 1:1 was observed $\left(\chi^{2}=6.24, d f=1, P<0.025\right)$. This effect was absent in the reciprocal crosses ( $s t / *$ parental females) (419:419) (table IV).

The validity of the assumption that flies were not differentially attracted to the collecting baits was tested in March 1989. Chromosomal frequencies estimated in samples of adult flies collected by means of banana baits and with rotting cladodes were not significantly different $\left(\chi^{2}=5.57, d f=2, P>0.05, N=189\right)$, pointing to the fact that flies were attracted to the baits irrespective of their karyotype.

\section{DISCUSSION}

Random mating is a general assumption in almost all models devised for population genetics purposes. In the $D$ buzzatii natural population of Arroyo Escobar, observed mating frequencies of second chromosome karyotypes did not depart significantly from those expected, giving support to the assumption of random mating. This result is in agreement with those obtained by Ruiz et al (1991) in a Spanish natural population of $D$ buzzatii.

However, our analysis is based on the assumption that the offspring of each wild inseminated female was fathered by a single male. Concerning this, female remating has often been observed in several species of Drosophila (Loukas et al, 1981; Markow, 1982). The frequency of multiple inseminations is dependent on environmental conditions such as population density (Turner and Anderson, 1983) and 
Table II. Observed numbers for the mating pairs in table I, when data were pooled for each second chromosome arrangement: a) $2 j$; b) $2 s t$ and c) $2 j z^{3}$. Random mating expected numbers are also shown.

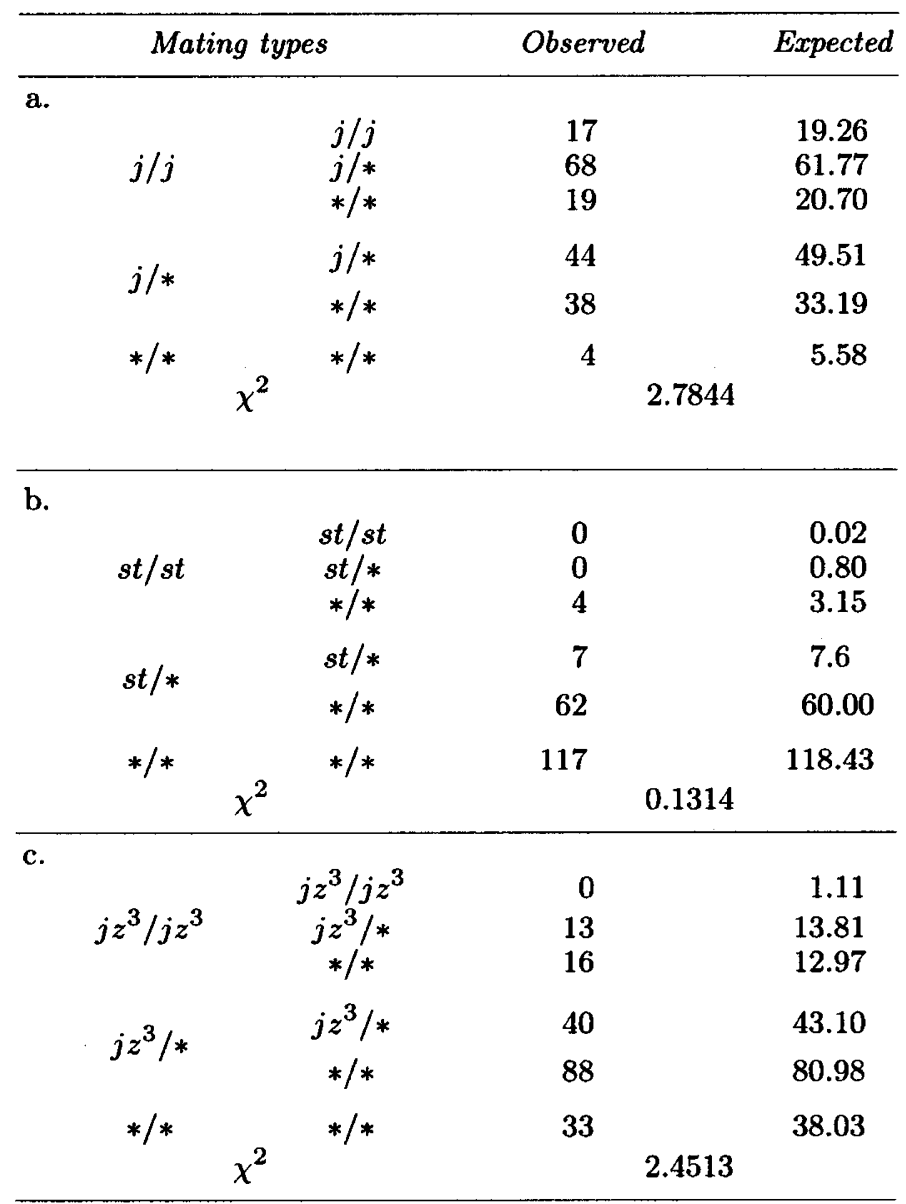

on the species considered (Markov, 1982). In the present work, flies were collected in mid-autumn, when their density and activity were low. Multiple insemination events in the present work were detected only in a few cases (less than $1 \%$ ), which were not considered in the analysis, because it was not possible to ascertain their parental karyotypes. Multiple insemination frequency was underestimated because some events might have been undetected; however, the close fitting of parental karyotype frequencies to Hardy-Weinberg expectations $\left(\chi^{2}=4.38, d f=2, P>0.05\right)$ gave support to the assumption that female remating should not contribute to bias our results.

In the segregation analysis, it should be noted that the departure from Mendelian segregation shown in table III was observed in culture vials in which the larval 
Table III. Comparison of observed and expected segregation patterns in the progeny of each mating class in the natural population of Arroyo Escobar.

\begin{tabular}{|c|c|c|c|c|c|c|}
\hline & Parents & Progeny & Observed & Expected & $\chi^{2}$ & $P$ \\
\hline \multirow{6}{*}{$j / j$} & \multirow{2}{*}{$J / j z^{3}$} & $j / j$ & 220 & 221.5 & \multirow{2}{*}{0.020} & \multirow{2}{*}{0.89} \\
\hline & & $j / j z^{3}$ & 223 & 221.5 & & \\
\hline & \multirow{2}{*}{$j / s t$} & $j / j$ & 51 & 42.0 & \multirow{2}{*}{3.857} & \multirow[t]{2}{*}{0.05} \\
\hline & & $j / s t$ & 33 & 42.0 & & \\
\hline & \multirow[t]{2}{*}{$j z^{3} / s t$} & $j / j z^{3}$ & 41 & 31.5 & \multirow[t]{2}{*}{5.730} & \multirow[t]{2}{*}{0.017} \\
\hline & & $j / s t$ & 22 & 31.5 & & \\
\hline \multirow{5}{*}{$j / s t$} & \multirow{3}{*}{$j / j z^{3}$} & $j / j$ & 33 & 32.5 & \multirow{3}{*}{14.062} & \multirow{3}{*}{0.003} \\
\hline & & $j / j z^{3}$ & 48 & 32.5 & & \\
\hline & & $\begin{array}{c}j / s t \\
j z^{3} / s t\end{array}$ & $\begin{array}{l}30 \\
18\end{array}$ & $\begin{array}{l}32.5 \\
32.5\end{array}$ & & \\
\hline & \multirow{2}{*}{$j z^{3} / j z^{3}$} & $i / i z^{3}$ & 29 & 26.0 & \multirow[b]{2}{*}{0.692} & \multirow[b]{2}{*}{0.41} \\
\hline & & $j z^{3} / j z^{3}$ & 23 & 260 & & \\
\hline \multirow{5}{*}{$j / j z^{3}$} & & $j / i$ & 40 & 43.0 & \multirow{3}{*}{0.837} & \multirow{3}{*}{0.66} \\
\hline & $j / j z^{3}$ & $j / j z^{3}$ & 84 & 86.0 & & \\
\hline & & $j z^{3} / j z^{3}$ & 48 & 43.0 & & \\
\hline & \multirow{2}{*}{$j z^{3} / j z^{3}$} & $j / j z^{3}$ & 47 & 40.5 & \multirow{2}{*}{2.086} & \multirow{2}{*}{0.15} \\
\hline & & $j z^{3} / j z^{3}$ & 34 & 40.5 & & \\
\hline
\end{tabular}

density was not under experimental control; thus, larval selection favoring $2 j$ or $2 j z^{3}$ cannot be ruled out. Crosses involving heterokaryotypes $2 j / s t$ and $2 s t / j z^{3}$ performed under controlled laboratory conditions yielded, in general, segregation patterns that did not depart from those expected, though a trend towards a deficiency of $2 s t$ in the progenies of males heterozygous for $2 s t$ was observed. This suggests the occurrence of gametic selection against 2st. Yet it should be noted that the $s t / s t$ homokaryotypic stock was obtained from only 4 independent chromosomes; thus the distortion observed might be the consequence of a homozygous residual genotype. Therefore, new experiments with $2 s t$ strains derived from more independent chromosomes are necessary to test the significance of this observation.

Nonetheless, the frequency of $2 s t$ in Arroyo Escobar is relatively low, the expected frequency of the cross $2 j / s t$ males $\times 2 s t / s t$ females would be less than $5.610^{-4}$, and, taking into account that the observed segregation distortion was nearly $60: 40$, it can be concluded that the present results do not necessarily invalidate the conclusions of our selection components analysis (Hasson et al, 1991). Furthermore, even though the expected frequency of crosses involving $2 s t$ heterozygous males in the population of Arroyo Escobar would be about 0.05, the observed segregation distortion was just 
Table IV. Comparison of observed and expected segregation patterns in the progeny of $2 s t$ heterokaryotypes obtained from laboratory stocks, crossed with homokaryotypes: $s t / s t, j / j$ and $z^{3} / j z^{3} . V$ indicates the mean viability value for each cross.

\begin{tabular}{|c|c|c|c|c|c|c|c|c|}
\hline & Parents & & Progeny & Obs & Expec & $V$ & $\chi^{2}$ & $P$ \\
\hline \multirow{3}{*}{ Female } & \multirow[b]{2}{*}{$s t / j$} & $s t / s t$ & $\begin{array}{c}s t / s t \\
j / s t\end{array}$ & $\begin{array}{l}82 \\
75\end{array}$ & $\begin{array}{l}78.5 \\
78.5\end{array}$ & 86.4 & 0.31 & 0.578 \\
\hline & & $\begin{array}{c}j / j \\
j z^{3} / j z^{3}\end{array}$ & $\begin{array}{c}s t / j \\
j / j \\
s t / j z^{3}\end{array}$ & $\begin{array}{l}61 \\
83 \\
64\end{array}$ & $\begin{array}{l}72 \\
72 \\
57\end{array}$ & 77.8 & 3.36 & 0.067 \\
\hline & \multirow{3}{*}{$s t / j$} & st/st & $\begin{array}{c}j / j z^{3} \\
s t / s t \\
j / s t\end{array}$ & $\begin{array}{c}50 \\
116 \\
166\end{array}$ & $\begin{array}{l}57 \\
141 \\
141\end{array}$ & $\begin{array}{l}70 \\
75.2\end{array}$ & $\begin{array}{l}1.72 \\
8.86\end{array}$ & $\begin{array}{l}0.190 \\
0.003\end{array}$ \\
\hline \multirow[t]{3}{*}{ Male } & & $\begin{array}{c}j / j \\
j z^{3} / j z^{3}\end{array}$ & $\begin{array}{c}s t / j \\
j / j \\
s t / j z^{3}\end{array}$ & $\begin{array}{l}57 \\
66 \\
59\end{array}$ & $\begin{array}{l}61.5 \\
61.5 \\
56\end{array}$ & 73.5 & 0.66 & 0.417 \\
\hline & & $s t / s t$ & $\begin{array}{c}j / j z^{3} \\
s t / s t\end{array}$ & $\begin{array}{l}53 \\
53\end{array}$ & $\begin{array}{l}56 \\
57.5\end{array}$ & 67.8 & 0.321 & 0.571 \\
\hline & \multirow{5}{*}{$s t / j z^{3}$} & & $j z^{3} / s t$ & 62 & 57.5 & 55.8 & 0.70 & 0.403 \\
\hline \multirow[t]{4}{*}{ Female } & & $j / j$ & $s t / j$ & 69 & 76 & & & \\
\hline & & $j z^{3} / j z^{3}$ & $\begin{array}{l}j z^{2} / 3 \\
s t / j z^{3}\end{array}$ & $\begin{array}{l}83 \\
90\end{array}$ & $\begin{array}{l}76 \\
78\end{array}$ & 85.6 & 1.29 & 0.256 \\
\hline & & $s t / s t$ & $\begin{array}{c}j z^{3} / j z^{3} \\
s t / s t\end{array}$ & $\begin{array}{l}66 \\
60\end{array}$ & $\begin{array}{l}78 \\
59.5\end{array}$ & 88.6 & 3.69 & 0.055 \\
\hline & & & $j z^{3} / s t$ & 59 & 59.5 & 58.2 & 0.01 & 0.920 \\
\hline \multirow[t]{4}{*}{ Male } & \multirow[t]{4}{*}{$s t / j z^{3}$} & $j / j$ & $s t / j$ & 71 & 78.5 & & & \\
\hline & & & $j z^{3} / j$ & 86 & 78.5 & 84.6 & 1.43 & 0.232 \\
\hline & & $j s^{3} / j z^{3}$ & $s t / j z^{3}$ & 50 & 54 & & & \\
\hline & & & $j z^{3} / j z^{3}$ & 58 & 54 & 65.3 & 0.59 & 0.442 \\
\hline
\end{tabular}

$4 \%$ (46:54). Therefore the deficiency of $2 s t$ progeny should be nearly $0.2 \%$. Once again, the assumption of absence of gametic selection seems to be acceptable.

Thus, the results reported herein give support to the conclusions reached in our previous paper. They demonstrate that the assumptions of lack of differential attraction of flies to the collecting baits and random mating can be accepted, and even the apparent weak gametic selection against $2 s t$ would not affect our previous results.

\section{ACKNOWLEDGMENTS}

The authors wish to thank to Dr OA Reig for helpful discussions and constructive criticisms of the manuscript. Dr A Fondevila suggested the importance of undertaking these studies. We are indebted to Dr G Tell and L M Giurfa for the French translation of the title and summary. The English language of this paper was improved by the revision of 
Mr FJ Dyzenchauz. The critical comments of two anonymous reviewers are also gratefully acknowledged. This work was supported by CONICET grant PIA 004-422/87 and by Universidad de Buenos Aires grant EX 030/87 awarded to OA Reig. EH is fellow of CONICET-Argentina, JJF is a fellow of the Universidad de Buenos Aires and JCV is a member of the Carrera del Investigador Ceintífico of CONICET.

\section{REFERENCES}

David J (1962) A new medium for rearing Drosophila in axenic conditions. Dros Inf Serv 36, 128

Endler JA (1986) Natural Selection in the Wild. Princeton University Press, New Jersey

Fontdevila A, Ruiz A, Ocãna J, Alonso G (1981) The evolutionary history of Drosophila buzzatii I. Natural chromosomal polymorphism in colonized population of the Old World. Evolution 35, 148-157

Hasson E, Vilardi JC, Naveira H, Fanara JJ, Rodriguez C, Fontdevila A, Reig OA (1991) The evolutionary history of Drosophila buzzatii XVI. Fitness components analysis in an endemic population from Argentina. $J$ Evol Biol 4, 209-225

Hedrick PW, Murray E (1983) Selection and measures of fitness. In: Genetics and Biology of Drosophila (Ashburner M, Carson HL, Thompson JN, eds) Academic Press, New York, vol 3c, 61-104

Loukas M, Vergini Y, Krimbas CB (1981) The genetics of Drosophila subobscura populations. XVIII. Multiple insemination and sperm displacement in Drosophila subobscura. Genetics 57, 29-37

Markow TA (1982) Mating systems of cactophilic Drosophila. In: Ecological Genetics and Evolution. Academic Press, Australia

Ruiz A, Fontdevila A, Santos M, Seoane M, Torroja E (1986) The evolutionary history of Drosophila buzzatii. VIII. Evidence for endocyclic selection acting on the inversion polymorphism in a natural population. Evolution 40,740-755

Ruiz A, Santos M, Barbadilla A, Quesada-Diaz JE, Hasson E, Fontdevilla A (1991) The evolutionary history of Drosophila buzzatii XVIII. Genetic variance for body size in a natural population. Genetics $128,739-750$

Turner ME, Anderson WW (1983) Multiple mating and female fitness in Drosophila pseudoobscura. Evolution 37, 714-723 
Book review

J Frézal, M-S Baule, T de Fougerolle (1991) Genatlas, $2^{\mathrm{e}}$, INSERM John Wiley Paris, $1013 \mathrm{p}, 500 \mathrm{~F}$

Il s'agit de la deuxième édition du catalogue des gènes humains extrait de la banque de données française Genatlas mise à jour jusqu'en janvier 1991.

Cet ouvrage recense tous les gènes et autres marqueurs génétiques localisés à ce jour sur les chromosomes de l'homme (6500 entrées, plus de 900 références). On dénombre 2500 entrées de plus que dans l'édition précédente parue en 1989 : c'est dire avec quel dynamisme progresse la connaissance du génome humain.

Pour l'homme, les premiers efforts informatisés de catalogage du génome remontent à 1962, avec la première édition de Mendelian Inheritance in Man (MIM) de Mc Kusick à Baltimore : 2118 entrées. Maintenant, seules les méthodes informatiques sophistiquées des banques de données constamment perfectionnées permettent de gérer le flux d'information qui, depuis cette date, s'est incroyablement accru et diversifié, en particulier avec les méthodes d'exploitation et de localisation des séquences d'ADN.

En fait, le catalogue Genatlas, que les spécialistes peuvent interroger «en ligne» sur leur ordinateur pour des renseignements complémentaires, ne constitue quelque sorte que la partie visible de l'iceberg.

Un chapitre avec 750 entrées traite des maladies héréditaires, les autres chapitres sont les 2 listes de gènes cartographiés (par ordre alphabétique de nom puis de symbole), les sites fragiles, les localisations sur les chromosomes, les catégories (antigènes, enzymes, hormones et récepteurs, etc).

Les médecins spécialistes des maladies héréditaires restent des utilisateurs ciblés privilégiés de Genatlas, dans la tradition de MIM, mais l'ouvrage intéresse aussi les nombreux chercheurs qui travaillent sur les variants génétiques et la carte chromosomique de l'homme, les biologistes en général et, évidemment, les généticiens des autres espèces pour lesquels les travaux faits sur l'homme sont si abondants et si détaillés qu'ils constituent une base de référence obligée eu égard aux homologies interspécifiques.

JJ Lauvergne

Génétique factorielle

CRJ

78352 Jouy-en-Josas cédex

France

BG Jamieson (1991) Fish evolution and systematics : evidence from spermatozoa. Camb Univ Press.

Cet ouvrage a le mérite d'explorer un domaine d'interface entre disciplines qui n'ont par ailleurs que peu de connexions. On ne peut que souhaiter que de telles 
entreprises se multiplient : ici, le spermatologiste inscrit les structures qu'il observe dans une trame historique et tente de comprendre leur sens évolutif; le systématicien y puise de nombreux caractères et de précieuses synapomorphies. Un effort louable a été fourni pour la partie systématique car l'auteur présente des phylogénies récentes de Deutérostomiens (pour la plupart des «Poissons»). Il semble particulièrement bienvenu d'avoir traité au début de l'ouvrage d'un certain nombre d'autres groupes de Deutérostomiens (en tant que groupes extérieurs), ce qui permet d'établir les fondements d'une analyse comparative des caractères spermatiques des «Poissons».

Cependant, on trouve malheureusement des incohérences, notamment concernant les rapports entre les cladogrammes et la classification présentée; ou encore entre les objectifs exposés de la «spermiocladistique» et les réelles applications (insuffisantes) qu'on en fait dans le livre (l'exploitation des caractères spermatiques à des fins phylogénétiques ne semble pas menée à fond). La polarisation des caractères, leur présentation dans les cladogrammes, les commentaires associés manquent souvent de clarté. Il est parfois impossible de savoir quel est l'état plésiomorphe d'un caractère, ce qui est grave (voir plus loin). Il n'y a pas de matrice de caractères, pas de procédure parcimonieuse pour la proposition d'une hypothèse phylogénétique alternative fondée sur les caractères spermatiques. Tout ceci laisse à penser que l'analyse cladistique des caractères (leur polarisation), la dynamique du test d'une hypothèse phylogénétique ne sont pas bien maîtrisées. Il faut ajouter cependant au bénéfice de l'auteur que les caractères spermatiques semblent hautement homoplasiques.

Tout d'abord, on se demande pourquoi l'auteur, présentant tout au long du livre ses caractères dans des cladogrammes, persiste à présenter une classification des Craniates comprenant toujours des groupes paraphylétiques (p 65, on trouve des Agnatha et des Crossopterygii!). On a l'impression, tout au long du livre, et notamment par le découpage des chapitres, que la phylogénie et la classification sont choses bien séparées. L'auteur serait-il mal à l'aise avec l'un des fondements de la cladistique selon lequel la classification doit.refléter la phylogénie et elle seule?

Venons-en aux maladresses de présentation des caractères. La démarche générale de l'auteur consiste à prendre un cladogramme (fondé sur des caractères morphoanatomiques) considéré comme vrai et à examiner l'évolution des caractères spermatiques selon les parentés exprimées par le cladogramme. C'est intéressant en soi car il s'agit là de savoir si les caractères spermatiques ont un sens phylogénétique, c'est-à-dire principalement s'ils sont souvent sujets à convergences ou à réversions, en d'autres termes, s'ils sont porteurs d'homoplasie. Mais on reste sur sa faim, car les cladogrammes morpho-anatomiques pris dans la littérature ont aussi des zones de fragilité, c'est-à-dire des regroupements peu argumentés, des synapomorphies douteuses. Or, lorsque les caractères spermatiques sont incompatibles avec les parentés du cladogramme, c'est-à-dire qu'ils requièrent un trop grand nombre de convergences ou de réversions dans le contexte phylétique imposé, on aimerait voir surgir un embryon de discussion de ce cladogramme. Une telle confrontation consisterait à comparer le nombre et la qualité des caractères morpho-anatomiques supportant le nœud et ceux des caractères spermatiques contredisant les précédents. Lorsque des caractères spermatiques cohérents entre eux pourraient aboutir à une proposition de parenté nouvelle, (par exemple p 150), cela n'est ni présenté sous forme d'arbre, ni analysé à l'aide d'une méthode de parcimonie, ni suffisamment développé dans le 
texte. Tout se passe comme si l'auteur refusait de tester les cladogrammes établis, ce qui serait pourtant intéressant. On aurait aimé voir, par exemple, des hypothèses de parentés alternatives fondées sur les caractères spermatiques et générées à l'aide d'une méthode de parcimonie, ou, à défaut, à l'aide d'une démarche parcimonieuse interactive telle que celle qu'autorise le logiciel Mac Clade. On a l'impression que l'auteur n'est pas franchement convaincu de la démarche cladiste tout en étant obligé de présenter des cladogrammes, ou alors qu'il ne maitrise pas bien celle-ci et qu'il a préféré rester prudent. Un certain nombre de maladresses de présentation dont on ne citera que quelques exemples plus loin laissent à penser que cette seconde interprétation est la bonne. Par exemple, p 150, fig 13.1, on se demande pourquoi l'auteur, plutôt que de mettre des points d'interrogation dans le cladogramme, n'a pas mis les Lepidogalaxoidei, groupe frère des Ostariophysi, puisqu'ils partagent l'apomorphie 47 . Si l'auteur considère 47 comme une convergence, il doit la symboliser autrement. De la même manière, les Galaxioidea devraient constituer le groupe frère des Argentinoidei sur la base de l'apomorphie 54 partagée. L'auteur semble ne pas vouloir s'engager à faire des propositions phylogénétiques et à les discuter, ce qui est dommage. Ainsi, dans le texte, on devrait avoir une proposition plus précise et plus explicite que «This arrangement [...] leads us to link argentinoids and salmonoids in the phylogeny more closely than usual». Le problème est le même p 46, fig 3.20. Par exemple, le caractère «acrosome lost» est perdu chez les salpidae et les pyrosomatida: pourquoi les branches n'ont-elles pas été jointes sur la base de cette apomorphie? Puisque ces groupes n'ont pas de point de branchement, pourquoi ne pourrait-on pas les brancher comme groupe frère du «basic didemnid» qui présente le même caractère et voir si cette proposition est parcimonieuse?

D'autre part, les figures arborescentes ne sont pas claires. Par exemple, p 103 fig 9.5, la répartition des états des caractères dans le cladogramme laisse perplexe. Pourquoi le caractère 8 n'est-il pas clairement dessiné comme une réversion puisqu'il est considéré comme une apomorphie des Verbébrés (Craniates moins Myxinoïdes) p 74 fig 6.2? Si l'auteur s'engage à indiquer sur les branches les plésiomorphies retenues, comme il le fait pour 8 chez les Actinistia, qu'en est-il alors pour les Choanata? Doit-on en déduire que 8 retrouve un état apomorphe chez les Dipneustes et les Tétrapodes puisqu'aucune indication n'est donnée pour 8 ? On ne sait pas à quoi correspond l'état plésiomorphe du caractère 8 ( $\mathrm{p} 65$, tableau 5.2, $\mathrm{p} 103$, fig 9.5), ni s'il existe un état dérivé par rapport à 8 . Difficile de comprendre alors l'analyse qui a été faite de ce caractère, d'autant plus qu'aucune matrice n'est présentée.

Lorsque les caractères spermatiques se révèlent homoplasiques compte tenu des branchements imposés, aucune procédure parcimonieuse n'est produite pour argumenter la nature de l'homoplasie : les convergences ou les réversions sont imposées sans arguments. Toujours dans la figure 9.5 de la p 103, le caractère 8 est dans son état apomorphe chez les Osteichthyes; il a subi une réversion chez les Chondrostei, les Sarcopterygii, puis a dû réverter une fois de plus chez les Choanata puisque le carré blanc « 8 » ne se retrouve ni chez les Dipneustes ni chez les Tétrapodes. N'est-il pas plus parcimonieux de considérer que 8 est dans son état plésiomorphe chez les Osteichthyes et acquiert chez les Cladistia, Neopterygii et Choanata leurs état dérivés? (On suppose que ceux-ci sont probablement distincts puisqu'ils ne sont même pas explicités dans l'arbre). En d'autres termes, l'auteur 
opte pour 3 réversions (dont un retour à l'état apomorphe) plutôt que pour 3 convergences dans ce seul arbre des Osteichthyes sans expliquer pourquoi. D'une manière générale, la répartition et le changement d'état des caractères dans les arbres ne sont pas clairement discutés. On ne trouve que quelques rares indications diluées dans le texte.

On a trop souvent du mal à comprendre à quel état plésiomorphe correspond un état apomorphe (même p 67-68, notamment rubriques «Nucleus», "Axoneme», «Egg», «Other features» (sic!)). Il est pourtant indiqué que les dispositions plésiomorphes sont celles trouvées chez Branchiostoma, mais il eût été bon de les rappeler (par exemple dans une matrice). D'autre part, l'analyse cladistique des caractères laisse perplexe car ceux-ci sont tous polarisés par rapport à Branchiostoma selon 2 états seulement : cela ne facilite pas les regroupements. Certains caractères spermatiques ne peuvent-ils pas présenter 3 états ou plus : un état dit apomorphe chez certains «Poissons» par rapport à Branchiostoma ne pourrait-il pas être plésiomorphe par rapport à un autre état présenté par un autre Vertébré? Après tout, cela peut être aussi dû à la nature même des caractères. Des matrices de caractères codés auraient été bienvenues, elles auraient nettement clarifié les choses, d'autant plus que les réversions sont présentées dans les arbres comme des «probable retained plesiomorphy». La construction d'un arbre à partir des seuls caractères spermatiques resterait pour le lecteur une entreprise difficile. Il est très surprenant de voir (p 74, fig 6.2) que 8, 6 et 4 sont dans leurs états apomorphes chez les Vertébrés et qu'ils sont plésiomorphes ches les Lamproies. Pourquoi ne sont-ils pas alors des apomorphies de Gnathostomes? Le texte de la p 80 laisse à penser qu'il doit y avoir une erreur dans la figure 6.2 de la $\mathrm{p} 74$.

Nous ne multiplierons pas les exemples. En dépit de l'effort remarquable de synthèse que représente cet ouvrage, on ne peut que regretter une mauvaise présentation des caractères polarisés (notamment absence de matrices), surtout dans les arbres. Il manque, de plus, une vraie confrontation entre les 2 corps de données et on note une réticence à proposer lorsque cela est possible des hypothèses phylogénétiques alternatives.

D'un point de vue spermatologique, cet ouvrage rassemble l'essentiel des informations disponibles sur l'ultrastructure des spermatozoïdes des Deutérostomiens. Y apparaissent d'ailleurs des données originales non publiées de l'auteur, comme l'existence d'«acrosome-like vesicle» au cours de la spermiogénèse de Gambusia affinis. En outre, un chapitre complet $(36 \mathrm{p})$ porte sur la conservation des gamètes des «Poissons", ce qui constitue une bonne et utile synthèse des données actuelles sur le sujet. La bibliographie comporte au total environ 500 références.

G Lecointre

R Billard

Laboratoire d'ichtyologie générale et appliquée, Muséum national d'histoire naturelle, Paris 
Calendar

Colloque international "Mathématiques et dynamique des populations»

(1-5 juin 1992, Université de Pau, France)

Rencontre entre biologistes et mathématiciens qui s'intéressent aux problèmes posés par les populations de gènes ou de cellules, aussi bien qu'à d'autres sujets de biologie mathématique des populations et d'épidémiologie. Le colloque réunira des spécialistes des mathématiques appliquées, des probabilistes et des statisticiens, des écologistes, des épidémiologistes et des spécialistes de biomédecine. Les sujets traités porteront aussi bien sur des théories mathématiques que sur l'analyse de modèles, l'exploitation de données tirées de la biologie cellulaire et moléculaire, de l'épidémiologie et la recherche sur le cancer.

International Meeting on Distance Analysis "Distancia' 92" (22-26 June 1992, Rennes, France)

The notions of distance, dissimilarity, and metrics arise in many scientific fields - as tools of analysis, as modes of representation, or targets for approximation. Moreover, these concepts are also used in different areas of mathematics, such as geometry, graph theory, probability theory/and mathematical statistics. The aim of the Distancia'92 meeting is to bring together all those who are interested in the study of distance, in order to exchange knowledge and experience, and to discuss new insights and techniques.

Information

Distancia 92, Université de Rennes II, 6 av G Berger, 35043 Rennes Cedex, France. Tel : 33993351 55; Fax : 33 993351 75. E Mail : distance @ UHB FR

$14^{\Theta}$ Réunion du Groupe de biologie et de génétique des populations

(16-18 septembre 1992; Toulouse, Université Paul Sabatier, France)

Organisateur : Nicolas Borot, CTR recherche polymorphisme humain; CHU Purpan; Avenue de Grande-Bretagne; 31059 Toulouse Cedex, France 
The joumal Genetics Selection Evolution publishes original articles, review articles, short notes, and letters to the editor in English and in French.

\section{Address manuscripts to :}

L Ollivier, INRA, Station dé Génétique Quantitative et Appliquée, 78352 Jouy-en-Josas Cedex, France.

\section{GENERAL PRESENTATION}

Three complete sets of the manuscript must be submitted. The manuscript must be typeset double-spaced, with margins of at least $3.5 \mathrm{~cm}$ at top, bottom and sides for editor's markings. Pages and lines per page must be numbered.

The layout should be arranged as follows : title page, summary in the language of the article, title and summa$r y$ in the other language (in French if the article is written in English and vice versa), introduction, materials and methods, results, discussion, acknowledgments, references, figure legends, tables, figures.

$N B$ : Please note the absence of full stops after all abbreviations.

\section{Title page}

The title page should include the following: title, authors' names, institution and department where the study was carried out, a short title for running headlines.

\section{Summaries}

The summaries (not more than 200 words in the language of the article and not exceeding 500 words in the other language) should be in a suitable form for abstracting services. Please avoid paragraphs and footnotes. References and undefined abbreviations are not allowed.

\section{Key words}

Up to 5 key words should be supplied, to assist the reader and facilitate information retrieval. Key words may be taken from the title, summary or text. Please avoid the plural form.

\section{References}

References in the text should include the author(s) name(s) and year of publication. When more than 2 authors are cited, the first author's name should appear followed by " $e t$ $a l$ ". A number of references cited together should be chronologically set out. Ex: Hartl et al (1986); Hartl and Reimoser (1988); Hartl (1990a).

In the bibliography, author's names are listed in alphabetical order, as follows: publications of a single author in chronological order, publications of the same author with one co-author in chronological order, publications of the author with more than one coauthor in chronological order. All entries in the reference list must correspond to references in the text and vice versa.

The spelling of authors' names and dates must be the same both in the text and in the reference list.

The titles of journals should be abbreviated according to the rules of Biosciences Information Service (Biosis). Words for which no abbreviation is given should be written in full.

Examples are given below of the layout and punctuation to be used in the references:

- article (please mention all the authors)

Lefort G, Ollivier L, Sellier P (1975)

Analyse du comportement et de la fréquence des gènes à effets visibles dans les fratries de germains et de demi-germains. Ann Génét Sél Anim 7, 365-377

- book

Charlesworth B (1980) Evolution in Age-Structured Populations. Cambridge Univ Press, Cambridge

- chapter in a book

Lemeunier F, David JR, Tsacas L, Ashbumer M (1986) The melanogaster species subgroup. In: The Genetics and Biology of Drosophila (Ashbumer M, Thompson JR, Carson HL, eds) Academic Press, London, vol 3, 147-256

- symposium or congress paper

Ollivier L, Bolet G (1981) La sélection sur la prolificité chez le porc: résultats d'une expérience de sélection sur dix générations. In: 13 Journées de la Recherche Porcine en France. Paris, 4-5 February 1981, Institut Technique du Porc, Paris, 261-267

\section{Illustrations (figures and tables)}

Illustrations ( 1 original and 2 good copies), should be numbered in Arabic numerals for tables. They should be referred to in the text by their number and their ideal position indicated in the margin. Lettering (symbols, numbers, etc) should not differ from figure to figure and should be of sufficient height and thickness to remain legible after reduction. Figures should be presented in the form of drawings on drawing or tracing paper or as sharp prints.

Photographs and half-tones should contain good contrast; line drawings should have a white background. Tables should not exceed 84 characters per line. The title of each table should be written above the corresponding table. Figure legends should appear on the same page and should be explicit so that the illustrations are comprehensible without reference to the text. Authors will be charged for colour prints. Figures and tables published elsewhere cannot be accepted without prior consent of the publisher and the author(s).

The editorial board maintains the option of returning, before evaluation, manuscripts to authors who do not comply with these recommendations. The author is advised to keep one manuscript and a set of figures.

\section{PROOFS AND REPRINTS}

Proofs will be sent to the author indicated on the title page. They should be carefully corrected and returned to the publisher within 48 hours of reception. If this period is exceeded, the galleys will be printed without the author's corrections. Should substantial changes in the original manuscript be requested, they will be made at the author's expense. Fifty reprints per contribution are available free of charge. An order form for additional reprints will accompany the proofs.

There is no page charge for text and black-and-white figures.

\section{COPYRIGHT}

As soon as the article is published, the author is considered to have transferred his rights to the publisher. Requests for reproduction should be sent to the latter. 


\section{INSTRUCTIONS AUX AUTEURS}

La revue Genetics Selection Evolution publie des articles de recherche, des articles de synthèse, des courtes notes, des tribunes libres, et des lettres à la Rédaction en anglais et en français.

Adresse pour la soumission des manuscrits :

L Ollivier, INRA, station de génétique quantitative et appliquée, 78352 Jouyen-Josas Cedex, France

\section{PRÉSENTATION GÉNÉRALE}

Le manuscrit est soumis en 3 exemplaires. Il est dactylographié en double interligne avec des marges d'au moins $3,5 \mathrm{~cm}$ en haut, en bas et sur les côtés pour les annotations de la rédaction. Les pages sont foliotées et les lignes de chaque page sont numérotées.

La présentation matérielle du manuscrit est la suivante : page de titre, résumé dans la langue de l'article, titre et résumé dans l'autre langue que celle de l'article (en anglais si l'article est rédigé en français et inversement), introduction, matériel et méthodes, résultats, discussion, remerciements, références, légendes des figures, figures, tableaux.

NB: On notera l'absence de points abréviatifs.

\section{Page de titre}

La page 1 du manuscrit indique le titre, le nom des auteurs, l'adresse de l'établissement où a été effectué le travail, un titre abrégé (titre courant).

\section{Résumés}

Les résumés (moins de 200 mots dans la langue de l'article et moins de 500 mots dans l'autre langue) doivent être présentés sous une forme adaptée à une exploitation par des services documentaires. Eviter les paragraphes, les renvois en bas de page. Les références bibliographiques et les abréviations non définies ne sont pas admises.

\section{Mots clés}

Cinq mots clés au plus doivent être fournis, afin de faciliter l'indexation de l'article dans les fichiers documentaires. Les mots clés peuvent provenir du titre, du résumé ou du texte. Eviter le pluriel.

\section{Références (bibliographiques)}

Dans le texte, les références sont citées par les noms d'auteurs suivis de l'année de publication. La citation des articles écrits par plus de 2 auteurs est faite par le nom du premier auteur suivi de «et al.». Les références citées ensemble dans le texte doivent être ordonnées chronologiquement. Ex. : Hartl et al (1986); Hartl et Reimoser (1988); Hartl (1990a).

La liste des références est organisée par ordre alphabétique des noms d'auteurs et par ordre chronologique pour un auteur donné. Si un auteur a écrit certains de ses articles avec un ou plusieurs coauteurs, l'ordre est le suivant : articles de l'auteur seul, classés par ordre chronologique; articles du même auteur avec un coauteur, classés par ordre chronologique; articles de l'auteur avec plus d'un coauteur, classés par ordre chronologique.

Toutes les références de la liste doivent correspondre à des références citées dans le texte et vice versa.

Les titres des périodiques doivent être abrégés selon les normes de Biosciences Information Service (Biosis). Ecrire en toutes lettres les mots pour lesquels aucune abréviation n'est donnée.

Le style et la ponctuation des références sont conformes aux modèles illustrés dans les exemples suivants :

- article (indiquer tous les auteurs)

Lefort G, Ollivier L, Sellier P (1975) Analyse du comportement et de la fréquence des gènes à effets visibles dans les fratries de germains et de demi-germains. Ann Génét Sél Anim 7, 365 377

\section{- ouvrage}

Charlesworth B (1980) Evolution in age-structured populations. Cambridge Univ Press, Cambridge

\section{- chapitre d'ouvrage}

Lemeunier F, David JR, Tsacas L, Ashburner M (1986) The melanogaster species subgroup. In : The genetics and biology of Drosophila (Ashburner M, Thompson JR, Carson HL eds) Acad Press, Londres, Vol 3, 147256

- communication (publiée) d'un colloque ou congrès

Ollivier L, Bolet G (1981) La sélection sur la prolificité chez le porc: résultats d'une expérience de sélection sur dix générations. In : 13e Journées de la recherche porcine en France. Paris, 45 février 1981, Institut technique du porc, Paris, 261-267

\section{Illustrations (figures et tableaux)}

Les illustrations (1 original et 2 bonnes copies) seront numérotées en chiffres arabes pour les figures, romains pour les tableaux, et indexées dans le texte par rappel de leur numéro. Indiquer dans la marge du manuscrit leur emplacement idéal. Le lettrage (symboles, chiffres, etc.) doit être uniforme pour toutes les figures et de taille suffisante pour rester lisible après réduction. Les figures seront présentées sous forme de dessins, tracés ou photographies. Les photos en demi-teinte devront être suffisamment contrastées; les photos de dessins ou tracés auront un fond blanc. Les tableaux ne doivent pas comporter plus de 84 caractères par ligne. Le titre de chaque tableau sera porté au-dessus du tableau correspondant. Les légendes des figures doivent être claires, permettant aux illustrations d'être compréhensibles par ellesmêmes; elles devront toutes être groupées sur une même feuille. Les figures en couleurs seront à la charge de l'auteur. Aucune reproduction d'une figure ou d'un tableau déjà publié ne peut être acceptée sans l'autorisation écrite de l'éditeur et des auteurs.

Le comité de rédaction se réserve le droit de renvoyer aux auteurs, avant toute lecture, les manuscrits qui ne seraient pas conformes à ces recommandations. Il demande que les auteurs conservent un exemplaire du manuscrit et des illustrations.

\section{ÉPREUVES D'IMPRIMERIE ET TIRÉS À PART}

Les épreuves d'imprimerie sont envoyées à l'auteur indiqué sur le manuscrit. Elles doivent être soigneusement corrigées et renvoyées à l'éditeur dans les 48 heures qui suivent la réception. En cas de retard, l'éditeur se réserve, après sa propre correction, de procéder à l'impression sans les corrections d'auteurs. Si l'auteur requiert des modifications importantes, il en supportera la charge financière. Les 50 premiers tirés à part sont gratuits. Un bon de commande pour des exemplaires supplémentaires est joint aux épreuves.

La publication des textes et des figures en noir et blanc est gratuite. DROITS DE REPRODUCTION

Dès que l'article est publié, l'auteur est réputé avoir cédé ses droits à l'éditeur. Les demandes de reproduction seront adressées à celui-ci. 


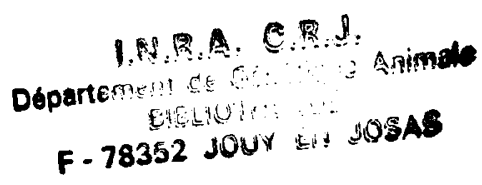

\section{Sommaire}

Déterminisme polygénique d'un caractère morphologique tout-ou-rien chez Euplotes (Ciliés Hypotriches). Signification évolutive de la variabilité morphologique naturelle chez les Ciliés

$J$ Génermont, C Demar, G Fryd-Versavel, H Tuffrau, M Tuffrau (Orsay,

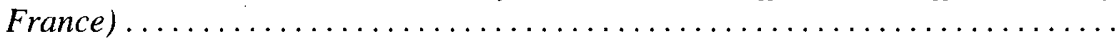

Inférence bayésienne des paramètres de dispersion de modèles mixtes univariates avec effets maternels : considérations théoriques

RJC Cantet, RL Fernando, D Gianola (Urbana, USA)................

Prise en compte des parentés pour l'évaluation des taureaux Limousins de monte naturelle

D Laloë, G Renand, J Sapa, F Ménissier (Jouy-en-Josas, France) .........

Influence du froid, du vent et de la pluie sur les dépenses énergétiques et la thermorégulation de sept types génétiques de brebis (en français)

JF Hocquette, M Vermorel, J Bouix (Saint-Genès-Champanelle, Castanet-

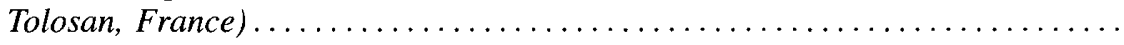

Réponse de la production d'œufs de poules albinos $\left(s^{\mathrm{a} 1}\right)$ et colorées $(S)$ à l'intensité lumineuse en poussinière

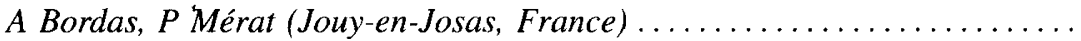

Le polymorphisme du deuxième chromosome de Drosophila buzzatii dans une population naturelle n'est pas associé à une sélection gamétique et n'affecte pas le mode d'accouplement

C Rodriguez, E Hasson, JJ Fanara, JC Vilardi (Buenos Aires, Argentine) .. $\quad 179$

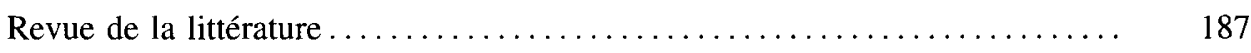

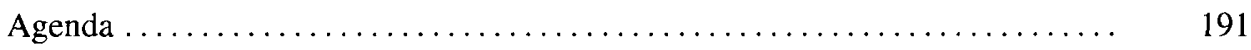

